\title{
The Energetic Cost of Reproduction and Its Effect on Optimal Life-History Strategies
}

\author{
Asta Audzijonyte ${ }^{1, *}$ and Shane A. Richards ${ }^{2}$ \\ 1. Institute for Marine and Antarctic Studies, University of Tasmania, Hobart, Tasmania 7001, Australia; 2. School of Natural Sciences, \\ University of Tasmania, Hobart, Tasmania 7001, Australia; and Oceans and Atmosphere, Commonwealth Scientific and Industrial Research \\ Organisation, Castray Esplanade, Hobart, Tasmania 7001, Australia
}

Submitted October 31, 2017; Accepted March 30, 2018; Electronically published June 29, 2018

Online enhancements: appendix, supplementary material.

ABSTRACT: Trade offs in energy allocation between growth, repro duction, and survival are at the core of life history theory. While age specific mortality is considered to be the main determinant of the op timal allocation, some life history strategies, such as delayed or skipped reproduction, may be better understood when also accounting for re production costs. Here, we present a two pool indeterminate grower model that includes survival and energetic costs of reproduction. The energetic cost sets a minimum reserve required for reproduction, while the survival cost reflects increased mortality from low postreproduc tive body condition. Three life history parameters determining age dependent energy allocation to soma, reserve, and reproduction are optimized, and we show that the optimal strategies can reproduce re alistic emergent growth trajectories, maturation ages, and reproduc tive outputs for fish. The model predicts maturation phase shifts along the gradient of condition related mortality and shows that increased harvesting will select for earlier maturation and higher energy alloca tion to reproduction. However, since the energetic reproduction cost sets limits on how early an individual can mature, an increase in fit ness at high harvesting can only be achieved by diverting most reserves into reproduction. The model presented here can improve predictions of life history responses to environmental change and human impacts because key life history traits such as maturation age and size, maxi mum body size, and size specific fecundity emerge dynamically.

Keywords: condition dependent mortality, fisheries induced evolu tion, indeterminate growth, maturation size, physiologically struc tured models, threshold reproduction cost.

\section{Introduction}

What determines the maturation and ultimate size of an organism with indeterminate growth (e.g., fish, amphibians, reptiles, and plants)? And, why do some individuals delay reproduction and instead invest in somatic growth? These

\footnotetext{
* Corresponding author; e-mail: asta.audzijonyte@utas.edu.au. ORCIDs: Audzijonyte, http://orcid.org/0000-0002-9919-9376.

Am. Nat. 2018. Vol. 192, pp. E000 E000. (C) 2018 by The University of Chicago. 0003-0147/2018/19204-58050\$15.00. All rights reserved. DOI: $10.1086 / 698655$
}

questions are at the core of life-history theory, and a number of models have been developed to help predict optimal energy allocation in different environments (e.g., Roff 1983; Kozłowski 1996; Charnov et al. 2001; Lester et al. 2004; Jørgensen and Fiksen 2006; Ejsmond et al. 2015). Life-history theory predicts that optimal allocation will be largely determined by age- and size-specific mortality regimes, which in turn determine maturation age, adult body size, and reproductive investment (Roff 2002). For example, experimental manipulation of fish mortality shows rapid evolutionary responses in growth, maturation, and reproductive traits (Reznick et al. 1997; Conover and Munch 2002; Uusi-Heikkilä et al. 2015). However, the optimal age-specific energy allocation to growth versus reproduction may also depend on costs associated with reproduction. Some organisms, such as broadcast spawning fish, may have a relatively low cost of reproduction, while others invest a lot of energy in reproductive behavior, such as migrations, mating behavior, or nest guarding (Lester et al. 2004; Kuparinen et al. 2012).

Reproductive costs can be divided into two components. First, the act of reproduction usually requires investment of energy that is not translated to offspring biomass. This investment includes the development and maintenance of reproductive organs and reproductive behavior. Lester et al. (2004) estimated energetic investment in reproduction in six marine fish species to be 1.2-2 times the gonado-somatic index, which simply compares the mass of gonads against somatic body weight. For example, Atlantic cod males lost $3 \%-19 \%$ of their body mass during experiments on mating behavior, and the weight loss was correlated to the number of courtship displays (Skjæraasen et al. 2010). Second, reproduction results in decreased body condition or reduced reserves, which may increase starvation mortality under low food availability or increase predation risk if weaker animals are less effective at escaping predation or spend more time foraging and are therefore more vulnerable to predation. In many cases, the costs associated with reproduction can be 
difficult to measure in wild populations, and the magnitude of these costs in different species still remains debated (Reznick 1985; Charnov et al. 2007). Yet there is good evidence that, at least in fish, postreproductive individuals often have low body condition, which in turn correlates with increased mortality (e.g., Dutil and Lambert 2000). Moreover, the decrease in postreproductive condition and mortality is often stronger in younger individuals. For example, in brook trout or pumpkinseed sunfish, postreproductive survival was as low as $10 \%-50 \%$ in the smallest individuals but $80 \%-$ $90 \%$ in the largest ones (Hutchings 1994; Bertschy and Fox 1999).

If reproduction entailed no investment or survival costs, then selection should favor early reproduction (Cole 1954). However, any survival cost due to reproduction means that "reproduction at ages of low fecundity involves an additional risk of failing to survive to ages of high fecundity, and selection may then favor delayed maturity" (Bell 1980, p. 59). It therefore seems that reproduction costs, both energetic and survival, are a key factor in explaining the diversity of life-history strategies. Moreover, if reproduction cost scales nonlinearly with size or age, this will also affect optimal resource allocation to reproduction and maturation size. For example, if the relative cost decreases with size, then it will favor delayed reproduction and, all else being equal, will lead to nonlinear increase of reproductive output with body size. In fish, this phenomenon has been recognized as the importance of "big old fat fecund female fish" (BOFFFs; Hixon et al. 2014), although it is rarely explicitly included in individual growth or population models. In fact, few growth models explicitly incorporate costs of reproduction (energetic or survival) and their interaction with maturation size altogether, and to our knowledge none have explored how these scale with body size. For example, biphasic growth models (e.g., Lester et al. 2004; Quince et al. 2008) include reproduction costs as a constant energy overhead, yet these costs do not determine maturation or spawn probability. Some state-dependent energy allocation models for Atlantic cod (Jørgensen and Fiksen 2006; Jørgensen et al. 2006) represent energetic reproductive cost through an energy threshold required for annual spawning migrations, which determines whether reproduction can occur at any given year. This approach, combined with stochastic food availability, was able to reproduce empirically known, but hard to model, skipped spawning events in individuals of low body condition. However, the model did not explore the possible scaling of this reproduction cost with body size and its consequences for life-history evolution. Kuparinen et al. (2012) incorporated a survival cost associated with reproduction into an individual-based evolutionary model of Atlantic cod. This cost increased postmaturation mortality rate and, as expected, favored later maturity at larger size. These studies suggest that explicit accounting for reproduc- tive costs is important to understand the evolution of lifehistory strategies in response to environmental change.

In order to better understand the importance of reproductive costs on life-history evolution, we present a general agestructured, two-pool, ectotherm growth model. The model follows the general principles of physiologically structured two-pool growth models (e.g., de Roos and Persson 2001; Giacomini et al. 2013; Ejsmond et al. 2015) and adopts some of the key principles of dynamic energy budget (DEB) theory (Kooijman 2000; Nisbet et al. 2012). We relax a commonly applied assumption of constant energy allocation between growth and reproduction (Heino and Kaitala 1999; Kooijman 2000), which brings our model closer to statebased or optimal allocation models (Kozłowski et al. 2004; Jørgensen and Fiksen 2006). Our model produces emergent individual growth, maturation, and reproduction based on size- and age-dependent food intake, metabolism, allocation rules, reproduction costs, and mortality. We explore the optimal life-history strategies under different scaling of energetic reproduction cost to body size, condition, and fishing mortality regimes and show that including a size-specific reproductive cost sets limits on the maturation size, which leads to more realistic emergent life-history strategies.

\section{The Model}

Our model describes individual female growth by tracking two biomass pools - nonreversible structural biomass $(S)$ and reversible biomass $(R)$ - and is comparable to commonly applied two-pool models (Persson et al. 1998). The $S$ pool includes bones, organs, and nonreversible muscle mass, whereas $R$ includes energy reserves and gonadal mass. Let $S_{a}$ and $R_{a}$ denote the mean mass ( $\mathrm{g}$ ) of $S$ and $R$ of an individual of age $a$ days, respectively. Individuals attempt to maintain an age-dependent $R: S$ ratio defined by

$$
\lambda_{a}=\lambda_{\min }+\left(\begin{array}{ll}
\lambda_{\max } & \lambda_{\min }
\end{array}\right) \frac{e^{r(a \bar{a})}}{1+e^{r(a \bar{a})}},
$$

where $\lambda_{\min }$ and $\lambda_{\max }$ are the minimum and maximum ratios, $\bar{a}$ is the age when the $R: S$ ratio exhibits an inflection point, and $r$ is the steepness of increase in allocation around the inflection point (table 1). A positive $r$ depicts increased allocation to $R$ with age, which describes a life history of initial somatic growth followed by a shift to reproduction (fig. 1).

Assimilated intake rate $\left(\mathrm{g}_{\text {day }}{ }^{1}\right)$ is given by $I(S)=$ $I_{0} S^{I_{1}}$ (assimilation efficiency is included in the mass-specific intake constant $I_{0}$ ). Here, we follow conventions of DEB theory and set $I_{1}=2 / 3$ (Kooijman 2000). Some, or all, of this intake is immediately allocated to a maintenance cost, which depends on both biomass pools: $C_{\mathrm{M}}(S, R)=c_{S} S+$ $c_{\mathrm{R}} R\left(\mathrm{~g}_{\text {day }}{ }^{1}\right)$. In line with $\mathrm{DEB}$, the maintenance cost has a scaling exponent of 1 but different mass-specific coefficients for the $S$ and $R$ pools, which, in the case of increasing 
Table 1: Parameters and their values

\begin{tabular}{|c|c|c|}
\hline & Symbol & Value \\
\hline \multicolumn{3}{|l|}{ Parameters: } \\
\hline Maximum age (years) & $a_{\max }$ & 20 \\
\hline Maximum $R / S$ & $\lambda_{\max }$ & 1.3 \\
\hline Minimum $R / S$ & $\lambda_{\min }$ & 0 \\
\hline Initial $S(\mathrm{~g})$ & $S_{0}$ & $1 /\left(1+\lambda_{0}\right)$ \\
\hline Initial $R(\mathrm{~g})$ & $R_{0}$ & $S_{0} \lambda_{0}$ \\
\hline Intake rate constant $\left(\mathrm{g} \mathrm{day}^{-1}\right)$ & $I_{0}$ & .1 \\
\hline Intake rate exponent & $I_{1}$ & .667 \\
\hline Maintenance cost of $S\left(\mathrm{~g}_{\text {day }}^{-1} \mathrm{~g}^{-1}\right)$ & $c_{\mathrm{S}}$ & .003 \\
\hline Maintenance cost of $R\left(\mathrm{~g}\right.$ day $\left.^{-1} \mathrm{~g}^{-1}\right)$ & $c_{\mathrm{R}}$ & .0003 \\
\hline Conversion efficiency to $S$ & $e_{\mathrm{S}}$ & .333 \\
\hline Conversion efficiency to $R$ & $e_{\mathrm{R}}$ & .9 \\
\hline Cost of reproduction $\left(C_{\mathrm{R}}\right)$ constant $\left(\mathrm{g} \mathrm{g}^{-1}\right)$ & $r_{0}$ & 6 \\
\hline$C_{\mathrm{R}}$ exponent & $r_{1}$ & .6 \\
\hline Weight to length conversion constant $\left(\mathrm{g} \mathrm{cm}^{-1}\right)$ & $l$ & $5,787.0$ \\
\hline Minimum mortality rate $\left(\right.$ year $\left.^{-1}\right)$ & $M_{\mathrm{P}, \min }$ & .2 \\
\hline Length based mortality rate when length $=0\left(\right.$ year $\left.^{-1}\right)$ & $M_{\mathrm{P}, \max }$ & 4.0 \\
\hline Steepness of the length based mortality rate $\left(\mathrm{m}^{-1}\right)$ & $z_{\mathrm{P}}$ & 8 \\
\hline Maximum condition dependent mortality rate $\left(\right.$ year $\left.^{-1}\right)$ & $M_{\mathrm{C}, \max }$ & 4.0 \\
\hline Steepness of the condition based mortality rate & $z_{\mathrm{C}}$ & 7 \\
\hline Maximum fishing mortality rate $\left(\right.$ year $\left.^{-1}\right)$ & $M_{\mathrm{F}, \max }$ & .3 \\
\hline Length at fishing mortality inflection (m) & $\bar{L}$ & .3 \\
\hline Steepness of the fishing mortality rate $\left(\mathrm{m}^{-1}\right)$ & $z_{\mathrm{F}}$ & 20 \\
\hline \multicolumn{3}{|l|}{ Optimized parameters: } \\
\hline Proportion of $R$ available for reproduction & $w$ & Optimized \\
\hline Age dependent $R$ allocation $\left(\right.$ day $\left.^{-1}\right)$ & $r$ & Optimized \\
\hline Age at inflection for $R$ allocation (day) & $\bar{a}$ & Optimized \\
\hline
\end{tabular}

$R / S$ with age, means that the overall mass scaling exponent is $<1$. If net intake rate $I_{\text {net }}(S, R)=I(S)-C_{\mathrm{M}}(S, R)$ is positive, then it is converted to $S$ and $R$ with efficiencies $e_{S}$ and $e_{R}$, so that energy to be allocated to either of the two pools is multiplied by the $e_{S}$ and $e_{R}$ values to get the final change in the pool biomass. Allocation of $I_{\text {net }}$ is such that the agespecific $R: S$ ratio $\left(\lambda_{a}\right)$ is either maintained or the $R: S$ ratio difference is minimized. This means that if $R_{a} / S_{a}<\lambda_{a}$, then all $I_{\text {net }}$ is channeled to $R$ until $\lambda_{a}$ is reached, and then $I_{\text {net }}$ is distributed between $R$ and $S$ to maintain the desired ratio. If $I_{\text {net }}<0$, then $S$ stays fixed and some (or all) of $R_{a}$ is used to cover the maintenance cost (or as much as possible), with the assumption that the conversion efficiency of the $R$ metabolic cost is again $e_{R}$ (so $1 \mathrm{~g}$ of $R$ mass will result in $0.9 \mathrm{~g}$ of mass to be used for maintenance costs; table 1).

Reproduction occurs annually, and the proportion of $R$ that is available for reproduction at the time of spawning is fixed through age at $w$ (but note that the $R: S$ ratio is age dependent). Thus, after spawning, the individual of age $a$ will have an $R$ pool equal to $(1 \quad w) R_{a}$, which can be used to mediate mortality due to starvation (see below). A novel feature of our model is a cost of reproduction $\left(C_{R}\right)$, which accounts for behaviors associated with reproduction (e.g., migration, territory establishment, or mate guarding). The reproduction cost is size specific and depends on the $S$ pool only as $C_{\mathrm{R}}(S)=r_{0} S^{r_{1}}(\mathrm{~g})$. Note that if $r_{1}<1$, then the relative cost of reproduction declines with size and can lead to delayed reproduction (fig. 2). If, at the time of reproduction, the proportion of the $R$ pool available for reproduction $(w R)$ is less than $C_{R}$, then reproduction cannot occur. Otherwise, the amount of spawn biomass produced is calculated as $F=w R \quad C_{R}$, and the $R$ pool is reduced to $(1 w) R$. If $C_{\mathrm{R}}$ is sufficiently high and intake is insufficient to replenish the $R$ pool since the previous reproduction, then the individual will not reproduce that year, which can lead to skipped reproduction as a life-history strategy. Thus, this cost sets the age at first reproduction, which, in addition to the $C_{\mathrm{R}}$ function, will also depend on rates of food uptake 


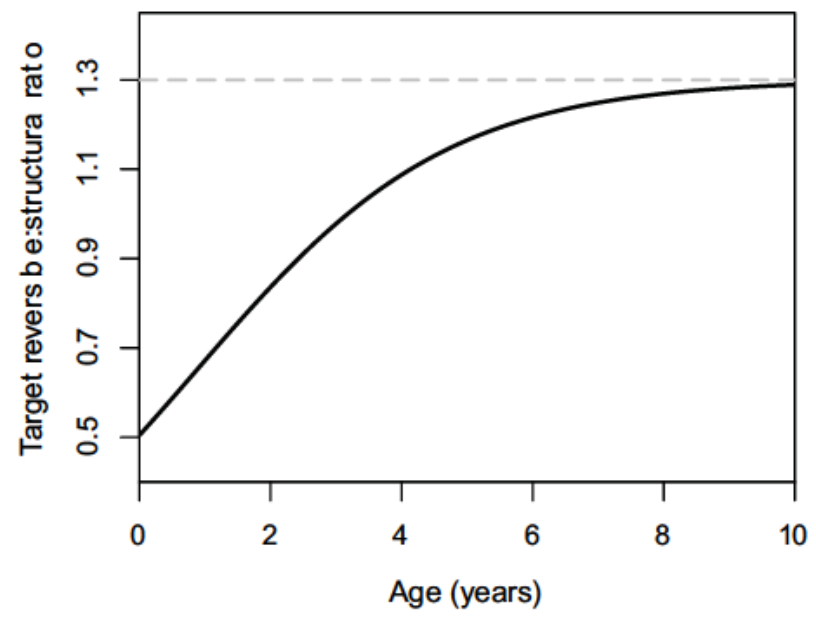

Figure 1: Example of a life history strategy where the target ratio of reversible to structural mass, $\lambda$, increases with age. Such strategy de scribes a species that delays reproduction in order to grow quickly when young.

and the $R / S$ allocation strategy defined by $\lambda_{a}$. We assume that reproduction cost is determined by an individual's size ( $S$ pool) rather than its condition because energetic cost of reproductive behavior is strongly determined by the activity cost, which is often scaled with an individual's length or structural size (e.g., Alexander 2003; Jørgensen and Fiksen 2006). The size of the $R$ pool might either interfere with activity functions (large gonads) or help them (high energy density), but quantitative information is limited and the possible role of the $R$ pool on energetic reproductive cost should be explored in a separate study.

Mortality has four additive components: condition, predation, fishing, and baseline. The first component includes mortality processes that are negatively influenced by animal condition (e.g., starvation), which we quantify as $R_{a} / S_{a}$. This ratio necessarily drops after spawning. The rate of conditiondependent mortality is given by $M_{\mathrm{C}}=M_{\mathrm{C}, \max } \exp \left(z_{\mathrm{C}} R_{a}\right)$ $S_{a}$ ), where $z_{\mathrm{C}}$ defines the steepness of how quickly mortality drops as $R / S$ increases (the exponential relationship means that condition related mortality in well-fed individuals is extremely small). Although we do not explicitly model stochasticity, low values of $z_{\mathrm{C}}$ can be used to describe environments where prey availability is variable and higher $R / S$ is required, on average, to avoid starvation mortality. The parameter $w$ determines the trade-off between allocation to reproduction versus future survival, and it becomes an important determinant of expected lifetime fitness.

The predation and fishing components of mortality are determined by fish length, which is determined by the $S$ pool, as $L(S)=(S / l)^{1 / 3}$, where $l$ is a proportionality constant similar to DEB's shape coefficient (see below). Here, we assume that larger individuals experience a lower risk of predation but a higher risk of fishing mortality. Fish of length $L(\mathrm{~m})$ experience rates of predation mortality as

$$
M_{\mathrm{P}}(L)=M_{\mathrm{P}, \min }+\left(M_{\mathrm{P}, \max } \quad M_{\mathrm{P}, \min }\right) e^{z \mathrm{p} L},
$$

where $z_{\mathrm{P}}$ determines the decline in mortality with size. Fishing mortality is modeled using a logistic selectivity function:

$$
M_{\mathrm{F}}(L)=\frac{M_{\mathrm{F}, \max }}{1+e^{\left.{ }^{{ }_{\mathrm{F}}(L \bar{L}}\right)}} .
$$

This continuous-time model was discretized using a daily time step, and reproduction was allowed to occur every 365 days, with the first potential reproduction on day 365 . The initial weight of all individuals is fixed at $1 \mathrm{~g}$, whereas $R_{0}$ and $S_{0}$ are set depending on the allocation strategy (see
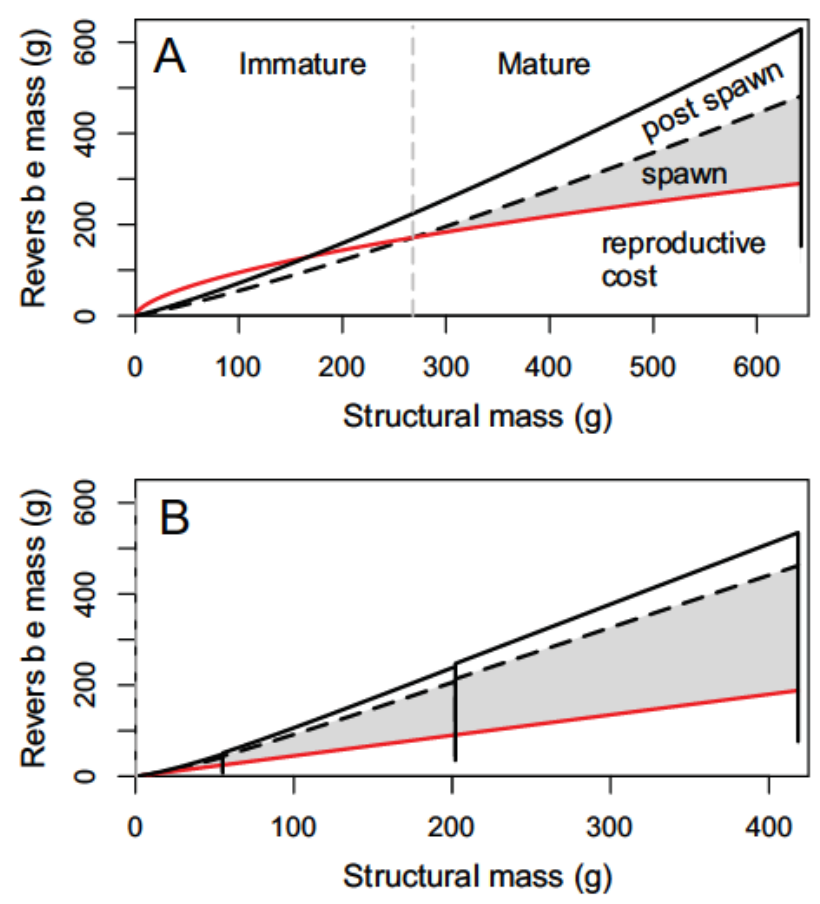

Figure 2: Schematic representation of how the age at maturation, spawn, and postreproductive conditions of individuals emerge in the model. The solid black line is the individual's reversible mass, and the dashed line is the reversible mass available for reproduction $(w R)$. Spawning occurs at the start of each year if available reversible mass surpasses the mass needed for reproduction, $C_{\mathrm{R}}$ (solid red line). Spawn ing events are indicated by a drop in reversible mass (vertical black lines). Two examples of optimal growth and reproduction are pre sented for the first 3 years of an individual's life: relative reproductive cost, $C_{\mathrm{R}} / S$, declines with structural mass $\left(r_{1}=0.6 ; A\right)$, and relative re productive cost is proportional to structural mass $\left(r_{1}=1 ; B\right)$. For the first example, spawning first occurs at age 3 years, whereas in the sec ond example, delayed reproduction is not optimal and spawning oc curs at age 1 year. 
below). We then track growth and survivorship of a single cohort up to age $a_{\max }$ (table 1).

\section{Selection of Life-History Parameters}

Three parameters define the life-history strategy, $\theta=$ $\{r, \bar{a}, w\}$, which determines how much energy is allocated to $R$ during development and how much $R$ is allocated to reproduction. We assumed that selection favors life-history strategy parameters that maximize expected lifetime spawning mass, equivalent to $R_{0}$, and calculated by summing up annual spawning masses weighted by the probability that the fish is alive at the time of spawning (fig. 3). Using $R_{0}$ (as opposed to the intrinsic rate of increase, or $r$ ) as a fitness measure assumes that density dependence happens early in life and affects all genotypes in the same way (Mylius and Diekmann 1995; Heino and Kaitala 1999), that there is no change in offspring fitness with parent's age (e.g., An-
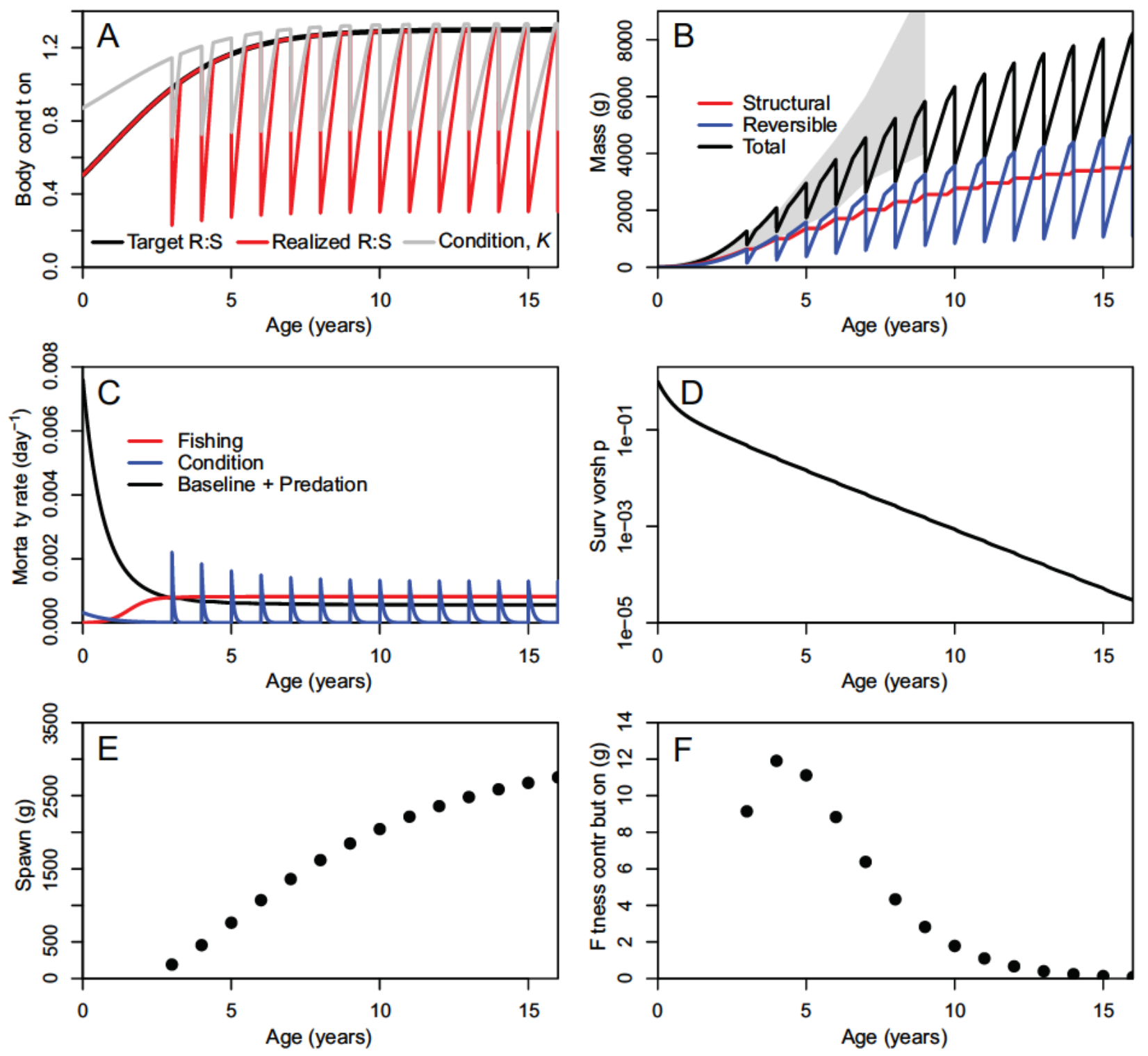

Figure 3: Optimal life history for the baseline scenario presented in table 1, where reproduction starts at 3 years. $A$, Target $R: S$ ratio, $\lambda_{a}$, and the realized ratio; also shown is the condition index, $K . B$, Growth dynamics, with the gray shaded region depicting the range of body masses typically reported for Baltic Sea cod; see table A1. C, Instantaneous daily mortality at age. $D$, Survival probability at age. $E$, Age dependent spawn production. F, Age dependent fitness contribution determined by spawn and survival. 
dersen et al. 2007), and that fitness associated with a strategy is independent of the strategy adopted by others (no frequency-dependent selection). The optimal combination of three life-history parameters for different reproduction cost and mortality parameters was found using the Solver add-in in Microsoft Excel 2016. The model code and the optimization file to explore the optimal life-history strategy under alternative parameter values are available in the supplementary material, available online. ${ }^{1}$ Because the fitness landscape is multipeaked or relatively flat, multiple starting parameter values must be used to ensure that the true fitness peak is found.

\section{Model Parameterization}

The model was parameterized for eastern/western Baltic Sea Atlantic cod (Gadus morhua), which has been heavily fished, lives in a highly seasonal environment where conditionrelated mortality is likely to be important, shows evidence of food limitation (Svedäng and Hornborg 2014), and trends toward earlier maturation age (Vainikka et al. 2009). Below we describe the selection and sensitivity analyses of model parameters.

Since intake is determined by $S$ only, the constant $I_{0}$ is higher than in models where intake is based on total weight and instead can be compared to the surface-area-specific maximum assimilation rate in DEB (ranging from 0.06 to $0.19 \mathrm{~g} \mathrm{~cm}^{2}$ day ${ }^{1}$; Kooijman and Lika 2014). The $I_{0}$ constant used here $\left(0.1 \mathrm{~g} \mathrm{~g}^{1} \mathrm{day}^{1}\right)$ leads to emergent daily intake of $0.5 \%-4 \%$ of total body weight in large-small individuals (assuming assimilation efficiencies of $0.7-0.8$, which are not included in the model explicitly), which is similar to those calculated for cod or whitefish in the wild conditions (Trudel et al. 2000) or those used in experimental conditions to achieve realistic growth rates (Kjesbu et al. 1996; Skjæraasen et al. 2009).

The average mass-specific maintenance cost of structure at $20^{\circ} \mathrm{C}$ in $\mathrm{DEB}$ is $20 \mathrm{~J} \mathrm{~cm}{ }^{3}$ day ${ }^{1}$ but in slow-growing vertebrates can be as low as $10 \mathrm{~J} \mathrm{~cm}^{3}$ day ${ }^{1}$ (Kooijman 2000). The environmental temperature of cod is closer to $10^{\circ} \mathrm{C}$, so the latter value seems more appropriate. This translates to $c_{S}$ of $0.003 \mathrm{~g} \mathrm{~g}^{1}$ day ${ }^{1}$, assuming $1 \mathrm{~g}$ of structure mass equals 3,000 J and $1 \mathrm{~cm}^{3}$ of wet weight is $1 \mathrm{~g}$ (Van der Veer et al. 2009). While reserves do not need maintenance in $\mathrm{DEB}$, the $R$ pool here includes reserves and gonads, and a small maintenance cost $\left(c_{R}\right)$ is used, set at $10 \%$ of $c_{S}$. These values give an emergent total maintenance cost of an adult individual at $40 \%-70 \%$ of its daily energy intake. In line with

\footnotetext{
${ }^{1}$ Supplementary material includes the Rmarkdown documents of the main code and an Excel file with the model optimization (Solver add-in required). Code that appears in The American Naturalist is provided as a convenience to the readers. It has not necessarily been tested as part of the peer review.
}

DEB, we assume that average activity costs (not related to reproductive behavior) are species specific (Nisbet et al. 2012) and can be included in the mass-specific maintenance cost constant $\left(c_{S}\right)$.

The efficiency of energy conversion into structure (specific structure costs) in DEB is $\sim 5,000-5,600 \mathrm{~J} \mathrm{~cm}^{3}$ (Van der Veer et al. 2009; Kooijman and Lika 2014), approximately corresponding to $1.6-1.8 \mathrm{~g}$ of energy required to build $1 \mathrm{~g}$ of structure. Much lower values were used in Jørgensen and Fiksen's (2006) model of Atlantic cod, with conversion efficiency to somatic and gonad tissue set at 0.08 and 0.4 , respectively. Since DEB includes other energydissipation fluxes, we assume an intermediate value for energy conversion efficiency to the $S$ pool $\left(e_{S}=0.33\right)$. Energy conversion to the $R$ pool does not incur cost in DEB, but a small inefficiency is applied here, since our $R$ pool includes both reserves and gonads, and $e_{\mathrm{R}}$ represents the cost of building and reabsorbing gonadal structures (in case of starvation).

The weight-length conversion constant, $l$, was parameterized to match estimated length-weight relationships given the $R: S$ ratio; its cubic root (using length in centimeters) is 0.18 and is close to the DEB shape coefficient used for fish (Kooijman 2000). The maximum energy density in DEB is calculated at $0.7-0.9 \mathrm{~g} \mathrm{~cm}^{3}$ (Kooijman and Lika 2014), which would correspond to an $R: S$ ratio of $0.7-0.9$, if $R$ did not include gonads. Here we assume the maximum $R: S$ ratio of 1.3; alternative values only had a minor effect on model outcomes. A body condition index was compared to empirical data on Baltic Sea cod condition and calculated as $K=W \times 100 \times L^{3}$, using total weight $(W=S+R, \mathrm{~g})$ and length $(L, \mathrm{~cm})$.

We assume that the relative cost of reproduction, $C_{\mathrm{R}}$, declines with size $\left(r_{1}<1\right)$, which is consistent with observation of relatively higher spawn in larger individuals (e.g., Hixon et al. 2014; fig. A1, available online) and more frequent skipped spawning in smaller individuals (Secor 2007). The relative energetic cost of behaviors that contribute to the cost of reproduction - such as mating, nest guarding, and spawning migrations - often decrease with size; for example, the exponent of spawning migration costs with weight for Atlantic cod adopted by Jørgensen and Fiksen (2006) is $\sim 0.3$. For the baseline scenario, we assume the reproductive cost exponent $r_{1}=0.6$ and constant $r_{0}=6$. These values gave the baseline maturation age of the Baltic Sea cod at 3 years and reproduction cost of $\sim 30 \%$ of total energy used in reproduction, broadly consistent with historical maturation age and values of energetic gonado-somatic index (Lester et al. 2004). We explore a range of alternative $r_{1}$ and $r_{0}$ values (see "Results").

For the baseline scenario, the steepness of the predation mortality function $\left(z_{\mathrm{P}}\right)$ was parameterized to get the instantaneous predation mortality of $\sim 0.55$ year ${ }^{1}$ at age 1 year 
(fig. A1; Köster et al. 2003), and we also assess a range of $z_{\mathrm{p}}$ values on optimal life history. For the baseline scenario, we set condition-dependent mortality to give $~ 5 \%$ monthly mortality of ages 5-20 during the first month after reproduction and conduct sensitivity analyses to different values. The midpoint of the fishing selectivity curve was set at $30 \mathrm{~cm}$, baseline $M_{\mathrm{F} \text {,max }}$ value of 0.3 year ${ }^{1}$ was used, and a range of values was explored (table 1).

\section{Results}

\section{Emergent Life History and Growth under the Baseline Scenario}

For the baseline scenario (table 1) of moderate fishing mortality $\left(M_{\mathrm{F}, \max }=0.3\right.$ year $\left.{ }^{1}\right)$, the model produced the emergent growth, maturation, fecundity, and condition values that were broadly consistent with empirical Baltic Sea cod observations (fig. 3; table A1, available online). The baseline size-dependent reproductive cost is presented in figure $2 \mathrm{~A}$, where $r_{1}=0.6$ implies that the relative cost declines with body size. The optimal strategy for this scenario was to increase the ratio of reversible to structural mass with age $\left(\lambda_{a}\right)$, reaching $\lambda_{\max }$ at about the age of 5 years (fig. 1). The optimal fraction of reversible mass allocated to reproduction $(w)$ was at 0.742 , and the optimal maturation age was 3 years, with the total weight at first spawn at 1,288 g. Body condition fluctuated between 0.7 and 1.3 (fig. $3 \mathrm{~A}$ ), which was similar to the values of $0.8-1.1$ observed in the Baltic Sea (Vainikka et al. 2009; see transformations in table A1) and values of 0.8-1.5 observed under experimental conditions (Vallin and Nissling 2000). Fishing and nonfishing sources of mortality were similar in magnitude for older individuals, and condition-dependent mortality was relatively high for the first few months after spawning (fig. 3C). Survivorship dropped quickly during the first year, and only about 1 in 1,000 individuals survived to age 10 years (fig. $3 D$ ), which is broadly consistent with the recorded ages of the Baltic Sea cod. The spawn production in young spawners was almost 10 times smaller than in old individuals (fig. $3 E$ ); however, due to higher survival, the highest contribution to population growth came from age 4 individuals (fig. $3 F$ ). Having established that the model is capable of predicting realistic life histories, we next investigated sensitivity of the life-history predictions to changes in three key processes: the sizedependent cost of reproduction, condition-dependent mortality, and fishing mortality (fig. 4).

\section{Size-Dependent Energetic Reproduction Cost as a Key Determinant of Maturation Age}

First, we varied the cost of reproduction by varying the scalar $r_{0}$ but fixed the power at $r_{1}=0.6$ (fig. $4 A, 4 B$ ). When the cost of reproduction was ignored $\left(r_{0}=0\right)$, the model predicted maturation at age 1 year, and the fraction of reversible mass was $w=0.752$. Increasing $r_{0}$ eventually resulted in maturity being delayed to 2 years and then 3 years (fig. 4A). On average, the optimal fraction of reversible mass allocated to reproduction increased with $r_{0}$; however, shifts in optimal allocation occurred at changes in maturation age (fig. 4A). Body mass at a given age was largely influenced by age at maturation; delays in maturation typically increased body mass because nonspawners do not decrease in mass. However, within each maturation age, the increases in reproductive allocation $w$ required for larger $r_{0}$ values decreased body mass in older individuals, because more energy was allocated to reproduction rather than left in reserves. Surprisingly, despite the cost of reproduction scalar $r_{0}$ having a strong effect on age and size at maturity, it had a much lesser effect on the body mass of 5- or 10-year-old individuals or an average individual in a population (fig. $4 B$ ).

Repeating the above analysis with $r_{1}=1$ (i.e., reproductive cost is proportional to structural mass) resulted in maturation always occurring at 1 year, provided $r_{0}$ was not too large. Delayed maturation did not occur in this case because the cost of reproduction was always below the allocated reversible mass for all structural masses (i.e., the red curve was always below the dashed curve in fig. $2 B$ ), implying that reproduction could occur at any size. These results indicate that for the model here, diminishing relative costs of reproduction with size (i.e., $r_{1}<1$ ) is necessary for delayed maturation to be an optimal life-history strategy.

\section{Survival Cost of Reproduction and Optimal Life History}

Survival cost of reproduction was simulated through condition-related mortality, which increased after spawning due to the drop in the $R: S$ ratio (fig. $3 A, 3 D$ ). To explore the sensitivity of optimal life-history strategies to conditionrelated mortality, we varied the steepness of the mortality function, $z_{\mathrm{C}}$, where higher values mean that mortality drops slower as condition improves and individuals of intermediate condition have higher mortality than if $z_{\mathrm{C}}$ were lower. In this way, higher $z_{\mathrm{C}}$ values could approximate less predictable environments, where higher reserves might be required to survive low food conditions.

The effect of $z_{\mathrm{C}}$ on optimal life history was nontrivial and resulted in rapid maturation phase shifts at intermediate $z_{\mathrm{C}}$ values (fig. $4 C, 4 D$ ). Low $z_{\mathrm{C}}$ meant overall higher mortality and shorter life span due to the high survival cost of reproduction; this selected for earlier maturation. However, to achieve early maturity required high investment in reproduction (higher $w$ ), which further decreased body condition after spawning. For intermediate $z_{\mathrm{C}}$, postspawning mortality was reduced and the optimal strategy switched to lower investment in reproduction, which delayed first re- 

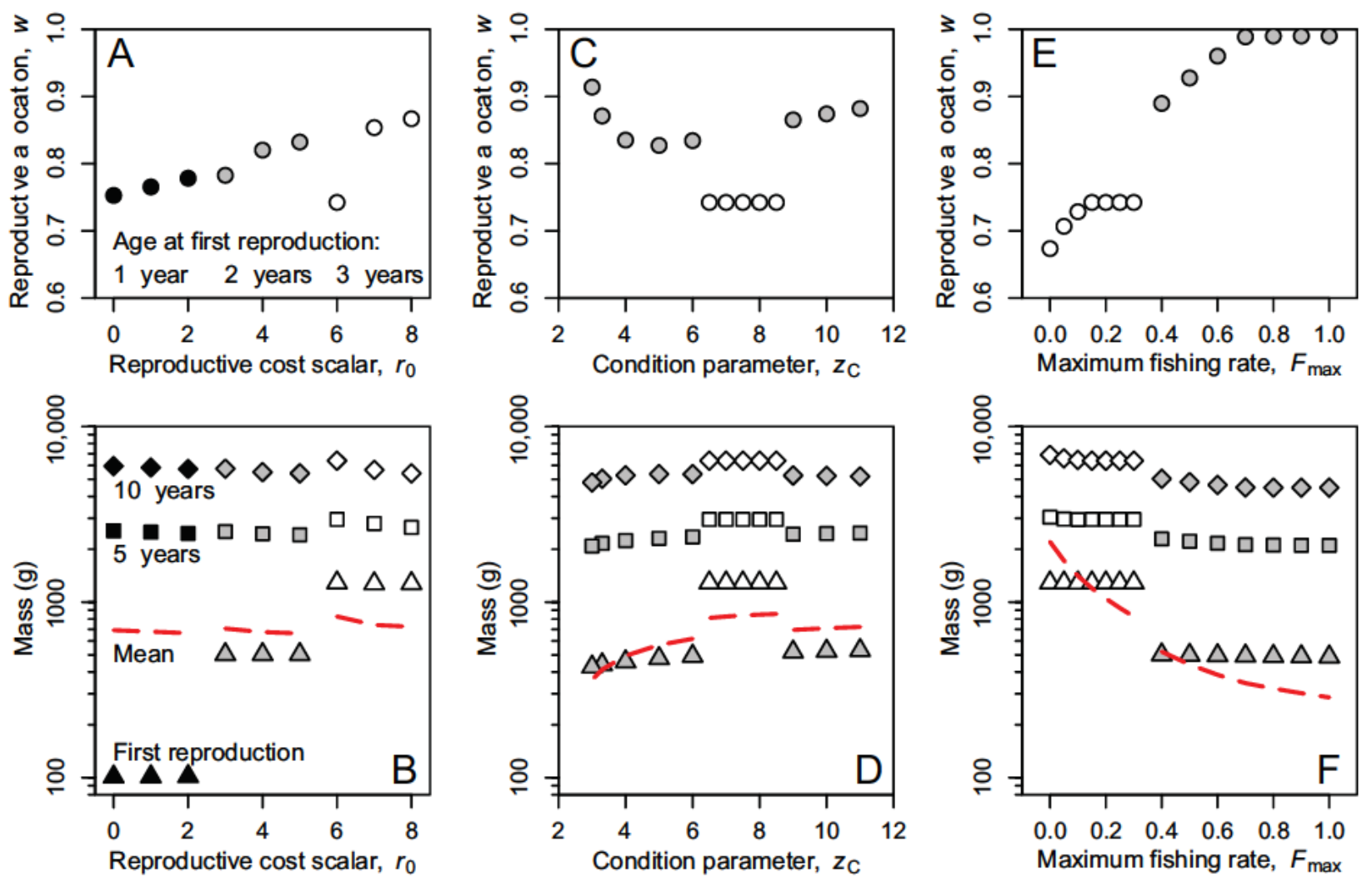

Figure 4: Optimal reserve allocation $(A, C, E)$ and resultant life history strategies and body mass $(B, D, E)$ under different energetic repro duction cost and mortality rates. Symbol colors indicate the emergent age at first reproduction: 1 year (black), 2 years (gray), and 3 years (white). $A, B$, Effect of the reproduction cost scalar $r_{0}$. $C, D$, Effect of condition mortality steepness $z_{\mathrm{C}} . E, F$, Effect of fishing mortality. $A, C$, and $E$ show the optimal fraction of reserves allocated to reproduction $(w)$. In $B, D$, and $F$, the total body mass is shown for first re production (triangles), at age 5 years (squares), and at age 10 years (diamonds). The mean body mass of all individuals in a population at the end of the year is indicated by red dashed lines.

production to age 3 years. However, when $z_{\mathrm{C}}$ was increased further and postspawning mortality was very low, highest fitness could be achieved by high investment in reproduction (because reserves were not essential for increased survival), which again brought forward the first reproduction to age 2 years. The mean body size of all individuals increased with higher $z_{\mathrm{C}}$ (low postspawn mortality) with a phase shift at intermediate-condition mortality values (fig. $4 D$ ).

\section{Optimal Life Histories and Fishing}

Changes in fishing mortality $M_{\mathrm{F}, \max }$ had a strong effect on optimal allocation to reproduction $w$ (fig. $4 E$ ), mean body size (fig. $4 F$ ), and maturation age. As fishing mortality increased and the survival and fitness contribution of older individuals dropped (fig. 5), fitness was optimized by increasing energy allocation to reproduction (higher $w$ ) and earlier maturation. Maturation occurred at age 3 years when $M_{\mathrm{F}, \max }<0.4$ year ${ }^{1}$, with a shift to 2 years and rapid increase in $w$ at higher fishing rates (fig. $4 E$ ). Because the energetic reproduction cost imposed constraints on the maturation age (sufficient energy had to be accumulated to meet the energy threshold), at very high fishing rates, maturation age could not be further reduced and fitness gains were made by greater investment in reproduction. When $M_{\mathrm{F}, \max } \geq 0.7$ year ${ }^{1}$, all energy in $R$ was allocated to reproduction (figs. $4 E, 5$ ), even at the cost of high postspawning mortality. This switch to earlier reproduction, and greater investment in reproduction, meant that increased fishing rates had a strong effect on reducing fish weights at age (fig. $4 F$ ) but a small effect on spawn produced by older individuals (fig. $5 B$ ). Importantly, mean fish body size in the population declined by nearly an order of magnitude from $2,203 \mathrm{~g}$ to $286 \mathrm{~g}$, as $M_{\mathrm{F}, \max }$ increased from 0 to 1.0 year ${ }^{1}$ (fig. $4 F$ ).

\section{Discussion}

Here, we have presented a novel and relatively general model of growth and reproduction for an indeterminate growth organism with annual reproduction. The key components of 

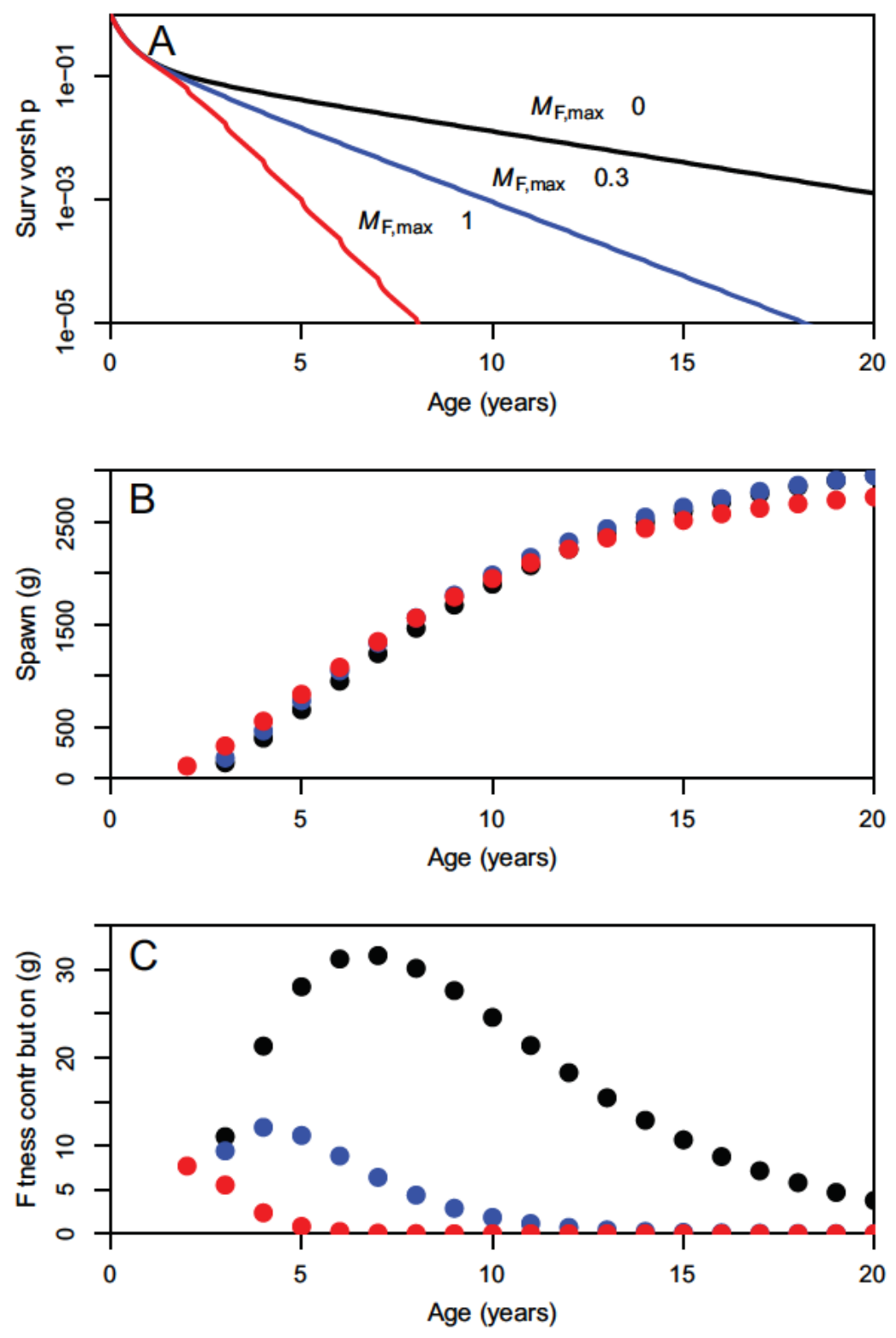

Figure 5: Effect of fishing on survival $(A)$, spawn at age $(B)$, and age specific fitness contribution $(C)$. The instantaneous fishing mortality of fully recruited individuals $\left(M_{\mathrm{F}, \max }\right)$ is 0 (black), 0.3 year $^{-1}$ (blue), and 1 year $^{-1}$ (red).

the model are a size-specific energetic reproductive cost, agedependent allocation to reversible and structural biomass pools, and condition-related mortality leading to a survival cost of reproduction. Together, these components determine the optimal age-dependent energy allocation to growth and reproduction. Importantly, rather than specifying maturation age or size, the model allows it to emerge dynamically in response to energy intake and allocation and size-specific reproduction cost. In this way, the model predicts delayed maturation at some mortality regimes and allows for the pos- sibility of skipped reproduction events if body condition at the time of reproduction is insufficient to account for the cost of reproduction. The trade-offs associated with energy allocation to growth, reserve, and reproduction and sizespecific reproduction costs lead to a large range of potential life-history strategies related to reproduction age and changes in reproductive investment throughout an individual's lifetime. Such dynamically emergent life-history models are important for predicting species responses to environmental changes, harvesting, and other human impacts. Below we 
summarize our key findings, compare them with prior studies, and provide suggestions for future work.

\section{Size-Dependent Energetic Reproduction Cost Sets Limits on Maturation Size}

Reproduction entails costs, both in terms of nonrecoverable energy expenditure required for successful reproduction to occur (energetic reproduction cost) and in the trade-off between current and future offspring production (survival cost of reproduction; Bell 1980; Heino and Kaitala 1999; Jørgensen et al. 2006). Larger size and better condition often brings decreased mortality, better success in competition for mates, increased fecundity, or relatively lower energetic cost of migration or other reproductive behavior (e.g., Folkvord et al. 2014). It is likely that below a certain size or condition, the cost of reproductive behavior is higher than the benefits of producing a small amount of offspring, setting a minimum energy threshold required for reproduction to occur at all. The existence of such a threshold in fish is suggested by the positive correlation between individual size and maturation probability in a given age group or correlation between low body condition at the early gonad development stage and probability of skipped spawning (Skjæraasen et al. 2009, 2012).

Here we formalize the energetic reproduction cost through a two-parameter size-dependent function. The function is necessarily only a crude approximation of the physiological and behavioral processes that affect reproduction but still provides a flexible and general way to explore the limits of life-history evolution and its consequences on populationlevel processes. For data-rich species, the cost could be considerably more specific, such as in the dynamic statedependent allocation model of Jørgensen and Fiksen (2006), where spawning migration cost of eastern Atlantic cod was parameterized for a specified migration distance, current velocity, and swimming activity (five parameters). The function could also include some dependence on the reversible biomass pool, which is ignored here. However, the function in Jørgensen and Fiksen (2006) still has a general shape of supralinear scaling with size, which means that reproduction becomes relatively cheaper for larger individuals. Such supralinear scaling, which is also applied here, is an important assumption that will have consequences on optimal life histories and is likely to vary among species. Our justification for supralinear scaling for cod-and possibly for other large fish with similar life histories - is based on (i) relative decrease in size-specific swimming activity cost, (ii) relative increase in mating or nest-guarding success in larger individuals (fig. A1), (iii) relatively higher reproductive output of large individuals and importance of BOFFFs, or (iv) positive maternal effects of larger individuals (Jørgensen and
Fiksen 2006; Rowe et al. 2008; see also fig. A1; Venturelli et al. 2010; Skjæraasen et al. 2012; Hixon et al. 2014).

The energetic reproductive cost function applied here sets a limit on maturation age and size. Our treatment of a reproduction cost is different from Lester et al. (2004), where the energetic cost is used as an energy-conversion scalar between spawn and energy used (energetic gonado-somatic index, ranging from 1.2 to 2.0 of the standard gonadosomatic index), rather than a size- or age-specific threshold determining whether reproduction will occur at all. This is an important distinction because only a threshold function will lead to the emergent dynamic maturation age or skipped reproduction events. In fish, skipped spawning is often observed empirically but rarely reproduced in models that predict life history (Jørgensen et al. 2006), unless an energetic reproduction cost threshold is applied and environmental stochasticity is included (Jørgensen and Fiksen 2006). Skipped spawning seems most common in species that live in highly seasonal environments or have energetically costly reproductive behavior (e.g., extensive spawning migrations; Rideout et al. 2005), but the phenomenon has also been demonstrated in nonmigratory species living in temperate lakes (Morbey and Shuter 2013), suggesting that energy threshold for reproduction might be a common feature, especially in capital breeders. Skipped spawning correlates with low condition (Skjæraasen et al. 2009) and results in higher somatic growth in skipped spawners compared to reproducing individuals (Skjæraasen et al. 2012; Folkvord et al. 2014), which suggests it is used as a strategy to increase lifetime fitness. Here, we did not focus on skipped spawning or include explicit environmental stochasticity or variability, but including a minimum energy threshold required for reproduction means that, under stochastic food availability, skipped spawning will be observed in ages and years where accumulated energy pool is insufficient to meet the energy threshold. Because the strategy of allocation to growth and reproduction is age dependent, younger individuals have relatively smaller reserve pools to cover the energetic reproduction cost. As a result, skipped spawning, just like in wild populations, will be more often observed in young individuals.

\section{Survival Cost of Reproduction and Condition-Related Mortality}

One common way to account for reproduction trade-offs in physiologically structured models is through decreasing sizespecific mortality. Since assimilated energy is partitioned between growth and reproduction, higher allocation to reproduction required for earlier maturation reduces growth and increases size-specific mortality (e.g., Andersen et al. 2007; Audzijonyte and Kuparinen 2016). This trade-off between reproduction and survival due to decreased growth was considered by Andersen et al. (2007) for their Baltic Sea 
cod model, which was used to explore optimal maturation size under fishing. The authors found that the optimal maturation size under high fishing mortality $\left(\mathrm{F} \sim 0.8\right.$ year $\left.{ }^{1}\right)$ was only $50 \mathrm{~g}$, which is about 10 times smaller than the maturation size obtained in our model for similar fishing mortality ( 500 g) or currently observed empirically ( 400$1,000 \mathrm{~g}$ for weight at age 3 ; see table A1). In fact, the smallest maturation size we observed in our model was at $100 \mathrm{~g}$ at maturation age of 1 year, but it was found only when reproductive costs were low ( $r_{0}$ of $0-2$; fig. $4 A$ ). To explain low optimal maturation weight under fishing in their model, Andersen et al. (2007) speculated that either insufficient time has passed to evolve to the optimal size or that other physiological or behavioral constraints, not included in the model, might be setting minimum limits on maturation size. Some of these additional constraints are represented in our study through the energetic cost of reproduction- and conditionrelated mortality (survival cost of reproduction) and explain the difference in emergent maturation weights in the two models.

Our findings suggest that accounting for survival cost of reproduction through reduced postmaturation growth - and hence increased predation mortality (as is the case in, e.g., Andersen et al. 2007 and many other modeling studies)does not represent the full range of reproduction-related costs. This is because size-specific mortality drops quickly in the first months of life, and survival cost of reproduction become too low when represented in this way. For example, Kuparinen et al. (2012) found that for Atlantic cod, the most realistic emergent life histories were produced when mature individuals had an additional instantaneous survival cost of reproduction of $0.1-0.15$ year ${ }^{1}$ applied through the entire postmaturation life span. This level of postreproductive mortality is consistent with parameters in Hutchings (2005) and appears on the lower end of some other empirically estimated costs (Hutchings 1994; Bertschy and Fox 1999).

Reproductive costs, both energetic and survival, will have important implications on optimal life histories under changing mortality regimes, such as harvesting or climate change. For example, it could be speculated that any negative consequences of fisheries-induced evolution on individual body size may be counteracted by earlier maturation and increased stock productivity. Yet if a reproduction cost sets limits on maturation size, it will constrain the ability of populations to adapt to increased mortality. Notably, under strong fishing pressure, there is a marked shift in both the age of individuals contributing the most spawn to the population, as well as in the absolute amount of lifetime reproductive output (fig. 5C). However, to address the effects of fishing on population productivity properly, our formulation of energetic and survival reproduction costs should be included in population or multispecies models that account for density dependence and dynamic predation mortality (e.g., for a case where earlier maturation led to decreased population growth rates due to increased predation, see Audzijonyte and Kuparinen 2016).

Our simulations also suggest that under high fishing mortality, fitness is increased through increasing allocation to reproduction from reserves, at the cost of immediate survival. This is generally consistent with empirical and experimental findings of increased gonado-somatic index (GSI) in intensively fished stocks (Sharpe and Hendry 2009; Audzijonyte et al. 2013), although empirically observed increase in GSI may be due to energy diversion from growth or from reserve. Broadly, our simulations suggested that when adult survival is low (fishing mortality is high) and net intake and size dependence of reproduction cost does not change, the life history evolves in the direction of semelparity, as all available energy is allocated to reproduction and postreproductive mortality is high. Of course, it is possible that the reproduction cost does not stay stable through time (as assumed here) but will itself evolve together with the energy-allocation strategy. Yet the presence of skipped reproduction, an expensive strategy, in a wide range of species suggests that, at least for a range of life-history strategies and reproductive behaviors, a minimum energetic threshold exists and cannot be further reduced.

\section{Model Limitations and Future Development}

The model presented here is able to capture key emergent life-history characteristics with only a few species-specific parameters. It introduces a concept of size-specific energetic reproduction costs as the energy threshold required for reproduction, which when scaled supralinearly with size, can give a mechanistic explanation for a wide range of observed phenomena in fish, such as longer spawn times, relatively higher reproductive output, and less frequent skipped spawning in older individuals. Our model can be readily extended to other indeterminate growing organisms or to investigate additional life-history aspects. For example, we currently do not include temperature effects, assuming that species either acclimate or modify their behavior to stay in optimal temperature. The model also currently assumes a capital breeding strategy (reproduction once per year), although it can be modified to allow for the constant or annual breeding under different seasonality assumptions (e.g., Ejsmond et al. 2015). It would also be informative to explore how the assumptions about the exponents of food intake and metabolic costs affect the emergent growth trajectories, given that different net exponents have been shown to lead to different optimal reproductive allocation in a model without a threshold reproductive cost (Boukal et al. 2014). We also suggest that the functional form and size-dependent scaling incorporated into the reproductive cost function be explored further. For example, high exponent $\left(r_{1}\right)$ and low 
constant $\left(r_{0}\right)$ might be suitable for $r$ strategists, where there is relatively little benefit of delaying reproduction to large size. Alternatively, a lower exponent value means that the relative spawn production will increase more at larger body size and may select for higher and early investment in growth, instead of early maturation. Finally, it would be informative exploring whether dependence of the energetic reproductive cost on both structural and reversible mass might lead to qualitatively different results. The main challenge here is to find suitable data to parameterize this function. Most empirical studies to date look at the change in condition or lipid content during spawning season, but size-dependent reproductive costs cannot be inferred from such data alone because it confounds both nonrecoverable costs (energetic reproduction cost) and the potential increase in reproductive allocation with age/size.

Importantly, our individual growth model does not include population- or community-level processes (e.g., resource competition), yet feedbacks via density- and frequencydependent interactions may also affect life-history evolution. For example, a model with density dependence acting at different life stages predicted two alternative evolutionarily stable life-history strategies of either early or late maturity (de Roos et al. 2006). Also, a multispecies marine model showed that, in contrast to expectations, when fishing selected for earlier maturation and smaller size, it led to decreased, rather than increased, population growth rates due to high predation pressure (Audzijonyte and Kuparinen 2016). The results from these studies and our own findings suggest that a worthwhile avenue of research is to include an explicit reproduction cost into more complex ecosystem models, particularly in the presence of fishing.

\section{Acknowledgments}

The authors would like to thank André de Roos, Ken Haste Andersen, Anssi Vainikka, editors Daniel I. Bolnick and Jürgen Groeneveld, and two anonymous reviewers for constructive comments on early versions of the manuscript. This study was supported by the Kone Foundation and Australian Research Council Discovery Grant DP170104240.

\section{Literature Cited}

Alexander, R. M. 2003. Principles of animal locomotion. Princeton University Press, Princeton, NJ.

Andersen, K. H., K. D. Farnsworth, U. H. Thygesen, and J. E. Beyer. 2007. The evolutionary pressure from fishing on size at matura tion of Baltic cod. Ecological Modelling 204:246 252.

Audzijonyte, A., and A. Kuparinen. 2016. The role of life histories and trophic interactions in population recovery. Conservation $\mathrm{Bi}$ ology 30:734 743 .
Audzijonyte, A., A. Kuparinen, and E. A. Fulton. 2013. How fast is fisheries induced evolution? quantitative analysis of modelling and empirical studies. Evolutionary Applications 6:585 595.

Bell, G. 1980. The costs of reproduction and their consequences. American Naturalist 116:45 76.

Bertschy, K. A., and M. G. Fox. 1999. The influence of age specific sur vivorship on pumpkinseed sunfish life histories. Ecology 80:2299 2313.

Boukal, D. S., U. Dieckmann, K. Enberg, M. Heino, and C. Jørgensen. 2014. Life history implications of the allometric scaling of growth. Journal of Theoretical Biology 359:199 207.

Charnov, E. L., T. F. Turner, and K. O. Winemiller. 2001. Reproduc tive constraints and the evolution of life histories with indetermi nate growth. Proceedings of the National Academv of Sciences of the USA 98:9460 9464.

Charnov, E. L., R. Warne, and M. Moses. 2007. Lifetime reproduc tive effort. American Naturalist 170:E129 E142.

Cole, L. C. 1954. The population consequences of life history phe nomena. Quarterly Review of Biology 29:103 137.

Conover, D. O., and S. B. Munch. 2002. Sustaining fisheries yields over evolutionary time scales. Science 297:94 96.

de Roos, A. M., D. S. Boukal, and L. Persson. 2006. Evolutionary re gime shifts in age and size at maturation of exploited fish stocks. Proceedings of the Roval Societv B 273:1873 1880.

de Roos, A. M., and L. Persson. 2001. Physiologically structured models from versatile technique to ecological theory. Oikos 94:51 71.

Dutil, J. D., and Y. Lambert. 2000. Natural mortality from poor con dition in Atlantic cod (Gadus morhua). Canadian Journal of Fish eries and Aquatic Sciences 57:826 836.

Ejsmond, M. J., Ø. Varpe, M. Czarnoleski, and J. Kozłowski. 2015. Seasonality in offspring value and trade offs with growth explain capital breeding. American Naturalist 186:E111 E125.

Folkvord, A., C. Jørgensen, K. Korsbrekke, R. D. Nash, T. Nilsen, and J. E. Skjæraasen. 2014. Trade offs between growth and repro duction in wild Atlantic cod. Canadian Journal of Fisheries and Aquatic Sciences 71:1106 1112.

Giacomini, H. C., D. L. DeAngelis, J. C. Trexler, and M. Petrere. 2013. Trait contributions to fish community assembly emerge from tro phic interactions in an individual based model. Ecological Model ling 251:32 43 .

Heino, M., and V. Kaitala. 1999. Evolution of resource allocation be tween growth and reproduction in animals with indeterminate growth. Journal of Evolutionary Biology 12:423 429.

Hixon, M. A., D. W. Johnson, and S. M. Sogard. 2014. BOFFFFs: on the importance of conserving old growth age structure in fishery populations. ICES Journal of Marine Science 71:2171 2185.

Hutchings, J. A. 1994. Age and size specific costs of reproduction within populations of brook trout, Salvelinus fontinalis. Oikos 70:12 20.

2005. Life history consequences of overexploitation to pop ulation recovery in Northwest Atlantic cod (Gadus morhua). Ca nadian Journal of Fisheries and Aquatic Sciences 62:824 832.

ICES. 2013. Report of the ICES Advisory Committee 2013. ICES Ad vice, 2012. Book 8. International Council for the Exploration of the Sea, Copenhagen.

Jørgensen, C., B. Ernande, Ø. Fiksen, and U. Dieckmann. 2006. The logic of skipped spawning in fish. Canadian Journal of Fisheries and Aquatic Sciences 63:200 211.

Jørgensen, C., and Ø. Fiksen. 2006. State dependent energy allocation in cod (Gadus morhua). Canadian Journal of Fisheries and Aquatic Sciences 63:186 199. 
Kjesbu, O. S., P. Solemdal, P. Bratland, and M. Fonn. 1996. Variation in annual egg production in individual captive Atlantic cod (Gadus morhua). Canadian Journal of Fisheries and Aquatic Sciences 53: 610620

Kooijman, S. A. L. M. 2000. Dynamic energy and mass budgets in bi ological systems. Cambridge University Press, Cambridge.

Kooijman, S. A. L. M., and K. Lika. 2014. Comparative energetics of the 5 fish classes on the basis of dynamic energy budgets. Lournal of Sea Research 94:19 28.

Köster, F. W., C. Möllmann, S. Neuenfeldt, M. Vinther, M. A. St. John, J. Tomkiewicz, H. H. Hinrichsen, B. MacKenzie, G. Kraus, and D. Schnack. 2003. Fish stock development in the Central Baltic Sea (1974 1999) in relation to variability in the environment. ICES Marine Science Symposia 219:294 306.

Kozłowski, J. 1996. Optimal allocation of resources explains inter specific life history patterns in animals with indeterminate growth. Proceedings of the Roval Societv B 263:559 566.

Kozłowski, J., M. Czarnołęski, and M. Dańko. 2004. Can optimal re source allocation models explain why ectotherms grow larger in cold? Integrative and Comparative Biology 44:480 493.

Kuparinen, A., D. C. Hardie, and J. A. Hutchings. 2012. Evolutionary and ecological feedbacks of the survival cost of reproduction. Evo lutionary Applications 5:245 255

Lester, N. P., B. J. Shuter, and P. A. Abrams. 2004. Interpreting the von Bertalanffy model of somatic growth in fishes: the cost of re production. Proceedings of the Roval Society B 271:1625 1631 .

Morbey, Y. E., and B. J. Shuter. 2013. Intermittent breeding in the ab sence of a large cost of reproduction: evidence for a non migratory, iteroparous salmonid. Ecosphere 4:1 18.

Mylius, S. D., and O. Diekmann. 1995. On evolutionarily stable life histories, optimization and the need to be specific about density de pendence. Oikos 74:218 224.

Nisbet, R. M., M. Jusup, T. Klanjscek, and L. Pecquerie. 2012. In tegrating dynamic energy budget (DEB) theory with traditional bio energetic models. Lournal of Experimental Biology 215:892 902.

Persson, L., K. Leonardsson, A. M. de Roos, M. Gyllenberg, and B. Christensen. 1998. Ontogenetic scaling of foraging rates and the dynamics of a size structured consumer resource model. Theoret ical Population Biology 54:270 293.

Pihl, L. 1982. Food intake of young cod and flounder in a shallow bay on the Swedish west coast. Netherlands Journal of Sea Re search 15:419 432 .

Quince, C., P. A. Abrams, B. J. Shuter, and N. P. Lester. 2008. Biphasic growth in fish. I: theoretical foundations. Iournal of Theoretical Bi ology 254:197 206.

Reznick, D. 1985. Costs of reproduction: an evaluation of the empir ical evidence. Oikos 257267

Reznick, D. N., F. H. Shaw, F. H. Rodd, and R. G. Shaw. 1997. Eval uation of the rate of evolution in natural populations of guppies (Poecilia reticulata). Science 275:1934 1937.

Rideout, R. M., G. A. Rose, and M. P. Burton. 2005. Skipped spawning in female iteroparous fishes. Fish and Fisheries 6:50 72.

Roff, D. A. 1983. An allocation model of growth and reproduction in fish. Canadian Journal of Fisheries and Aquatic Sciences 40:1395 1404.

2002. Life history evolution. Oxford University Press, Oxford.

Rowe, S., J. A. Hutchings, J. E. Skjæraasen, and L. Bezanson. 2008. Morphological and behavioural correlates of reproductive success in Atlantic cod Gadus morhua. Marine Ecology Progress Series 354:257 265 .
Secor, D. H. 2007. The year class phenomenon and the storage effect in marine fishes. Journal of Sea Research 57:91 103.

Sharpe, D. M., and A. P. Hendry. 2009. Life history change in com mercially exploited fish stocks: an analysis of trends across studies. Evolutionary Applications 2:260 275.

Skjæraasen, J. E., J. Kennedy, A. Thorsen, M. Fonn, B. N. Strand, I. Mayer, and O. S. Kjesbu. 2009. Mechanisms regulating oocyte re cruitment and skipped spawning in Northeast Arctic cod (Gadus morhua). Canadian Journal of Fisheries and Aquatic Sciences 66:1582 1596.

Skjæraasen, J. E., J. J. Meager, and J. A. Hutchings. 2010. A cost of re production in male Atlantic cod (Gadus morhua). Canadian Jour nal of Zoology 88:595 600 .

Skjæraasen, J. E., R. D. M. Nash, K. Korsbrekke, M. Fonn, T. Nilsen, J. Kennedy, K. H. Nedreaas, et al. 2012. Frequent skipped spawning in the world's largest cod population. Proceedings of the National Academy of Sciences of the USA 109:8995 8999 .

Svedäng, H., and S. Hornborg. 2014. Selective fishing induces density dependent growth. Nature Communications 5, doi:10.1038/ncomms 5152.

Trudel, M., A. Tremblay, R. Schetagne, and J. B. Rasmussen. 2000. Estimating food consumption rates of fish using a mercury mass balance model. Canadian Journal of Fisheries and Aquatic Sciences 57:414 428 .

Uusi Heikkilä, S., A. R. Whiteley, A. Kuparinen, S. Matsumura, P. A. Venturelli, C. Wolter, J. Slate, et al. 2015. The evolutionary legacy of size selective harvesting extends from genes to populations. Evo lutionary Applications 8:597 620 .

Vainikka, A., A. Gardmark, B. Bland, and J. Hjelm. 2009. Two and three dimensional maturation reaction norms for the eastern Baltic cod, Gadus morhua. ICES Journal of Marine Science 66:248 257.

Vallin, L., and A. Nissling. 2000. Maternal effects on egg size and egg buoyancy of Baltic cod, Gadus morhua: implications for stock struc ture effects on recruitment. Fisheries Research 49:21 37.

van der Veer, H. W., J. F. Cardoso, M. A. Peck, and S. A. Kooijman. 2009. Physiological performance of plaice Pleuronectes platessa (L.): a comparison of static and dynamic energy budgets. Journal of Sea Research 62:83 92.

Venturelli, P. A., C. A. Murphy, B. J. Shuter, T. A. Johnston, P. J. van Coeverden de Groot, P. T. Boag, and W. C. Leggett. 2010. Mater nal influences on population dynamics: evidence from an exploited freshwater fish. Ecology 91:2003 2012.

\section{References Cited Only in the Online Appendix}

Dunlop, E. S., M. Heino, and U. Dieckmann. 2009. Ecogenetic mod eling of contemporary life history evolution. Ecological Applica tions 19:1815 1834.

Fey, D. P., and T. B. Linkowski. 2006. Predicting juvenile Baltic cod (Gadus morhua) age from body and otolith size measurements. ICES Journal of Marine Science: Journal du Conseil 63:1045 1052.

Isomaa, M., V. Kaitala, and J. Laakso. 2014. Precautionary manage ment of Baltic Sea cod (Gadus morhua callarias) under different en vironmental noise and harvesting strategies. Boreal Environment Research 19:39 50. 